\title{
Autophagy and ER stress in LPS/GalN-induced acute liver injury
}

\author{
TING SHI ${ }^{1}$, WEIFANG SONG ${ }^{1}$ and RUILING $\mathrm{XU}^{2}$ \\ ${ }^{1}$ Department of Pathophysiology, Fenyang College of Shanxi Medical University, Fenyang, Shanxi 032200; \\ ${ }^{2}$ Department of Pathophysiology, Shanxi Medical University, Taiyuan, Shanxi 030001, P.R. China
}

Received September 6, 2016; Accepted June 8, 2017

DOI: $10.3892 / \mathrm{mmr} .2017 .7409$

\begin{abstract}
Numerous mechanisms and factors have been implicated in liver damage; however, the involvement of autophagy and endoplasmic reticulum (ER) stress during early-stage liver injury remains to be fully elucidated. The present study was conducted to determine the expression of autophagy and ER-stress-associated proteins in hepatic tissues following injury. A murine model of liver injury was induced by intraperitoneally injecting lipopolysaccharide (LPS) and D-galactosamine (GalN), and control mice were similarly injected with normal saline. The gross and histological appearance of the liver was examined, serum alanine aminotransferase (ALT) levels were detected, and protein expression evaluated via Western blot analysis. Co-administration of LPS and GalN effectively induced liver injury in mice, with severe liver damage manifesting at $6 \mathrm{~h}$ following induction. Furthermore, upregulation of autophagy-associated proteins, including microtubule-associated protein 1 light chain 3 (MAP1 LC3 or, briefly, LC3-II) and Beclin 1, were detected $3 \mathrm{~h}$ following liver injury. Consistently, the expression of the ER stress factor CCAAT/enhancer binding protein (C/EBP)-homologous protein decreased after $1 \mathrm{~h}$, but increased $3 \mathrm{~h}$ after liver damage. Autophagy and ER stress occurred in early-stage liver injury induced by LPS-GalN administration in mice. Induction of autophagy may act as a compensatory mechanism during early liver injury.
\end{abstract}

\section{Introduction}

Acute liver injury or acute liver failure is a rare, but life-threatening, disorder associated with high morbidity and mortality (1). Extensive hepatocyte cell death is typically observed in the pathogenesis of acute liver injury (2). The creation of animal models with acute liver injury through exposure to lipopolysaccharide (LPS) and D-galactosamine (GalN) is an extensively

Correspondence to: Dr Ting Shi, Department of Pathophysiology, Fenyang College of Shanxi Medical University, No. 16 Yingxiongbeilu, Fenyang, Shanxi 032200, P.R. China

E-mail: 65319952@qq.com

Key words: acute liver injury, lipopolysaccharide, D-galactosamine, autophagy, CCAAT/enhancer binding protein (C/EBP)-homologous protein, endoplasmic reticulum stress applied approach in rodents; the efficacy of combined treatment with these agents in inducing hepatotoxicity is greater as compared with that induced by either drug alone $(3,4)$. However, the pathogenesis of liver damage induced by co-administration of LPS and GalN has not yet been fully elucidated.

Hepatocyte apoptosis and necrosis is a predominant feature during acute liver injury (5). In addition to apoptosis and necrosis, it has previously been demonstrated that autophagy may be induced in hepatocytes upon LPS/GalN-induced liver injury (6). Autophagy is known to exhibit a pivotal role in maintaining cellular homeostasis through self-digestion of long-lived proteins or damaged organelles under stimuli (7). During induction of autophagy, cytosolic components are encapsulated in a phagophore, which forms a double-membrane-bound vesicle termed an autophagosome. This newly formed autophagosome fuses with a lysosome, allowing degradation of encapsulated products, whereby molecules and proteins may be recycled to improve cell survival. It has been suggested that autophagy not only modulates normal liver function, but also participates in the pathogenesis of various liver disorders (8). A previous study demonstrated that autophagy is closely associated with endoplasmic reticulum (ER) stress during the development of steatohepatitis in diabetic mice (9). The expression of ER stress-signaling components, including CCAAT/enhancer binding protein (C/EBP)-homologous protein (CHOP; a pro-apoptotic transcription factor), are positively associated with autophagic activity in liver tissues (9). In accordance with the results from the present study, it has previously been demonstrated that CHOP contributes to hepatocyte death during acute liver injury (10).

Given that ER stress is a potent trigger for induction of autophagy $(11,12)$, it is possible that acute liver injury may lead to ER stress, which subsequently activates autophagy. To investigate this hypothesis, a murine model of acute liver injury was created by intraperitoneally injecting LPS and GalN. Expression of autophagy and ER-stress-associated proteins were examined at indicated time points following the onset of acute liver injury.

\section{Materials and methods}

Reagents. Lipopolysaccharide was obtained from Sigma-Aldrich; Merck KGaA (Darmstadt, Germany). GalN was provided by Chongqing Medical University, (Chongqing, China). Anti-microtubule-associated protein 1 light chain (LC)-3, anti-Beclin-1, anti-CHOP, and anti- $\beta$-actin primary 
antibodies were all purchased from Cell Signaling Technology, Inc., (Danvers, MA, USA). Horseradish peroxidase (HRP)-conjugated secondary antibodies were purchased from Wuhan Boster Biological Technology Co., Ltd (Wuhan, China).

Animals and experimental assignment. A total of 34 male Kunming mice (age, 8-10 weeks; weight, $25 \pm 5 \mathrm{~g}$ ) were obtained from the Laboratory Animal Center, Shanxi Medical University (Taiyuan, China) [SCXK (Jin) 2009-0001]. Mice were housed together and acclimatized at $18-26^{\circ} \mathrm{C}$ at a constant humidity of $65-80 \%$ under standard lighting conditions (12/12 h light/dark) for 1 week and then randomly assigned to 4 groups: Control $(\mathrm{n}=8), 1$-h liver injury $(\mathrm{n}=8), 3$-h liver injury $(\mathrm{n}=8)$, and 6-h liver injury $(n=10)$. Liver injury in test mice was induced by an intraperitoneal injection of $0.2 \mathrm{ml}$ normal saline containing $5 \mu \mathrm{g}$ LPS and $0.02 \mathrm{~g}$ GalN, whereas controls were administered $0.2 \mathrm{ml}$ normal saline by intraperitoneal injection. Mice had free access to food and water. This study was approved by the ethics committee of Shanxi Medical University.

Sample collection. At 1, 3, or 6 h following LPS/GalN injection, blood samples were collected from the eye vein into heparinized tubes, and mice were sacrificed via cervical dislocation. The left lobe of the liver was removed from each mouse and tissue samples were processed by fixation in 10\% formaldehyde solution for 24-48 $\mathrm{h}$ at room temperature, washed with sterile normal saline, wrapped with sterile tinfoil, frozen in liquid nitrogen, and stored at $-70^{\circ} \mathrm{C}$ in a refrigerator until further analysis.

Histological examination. For histological examination, tissue samples were prepared for hematoxylin and eosin (HE) staining; briefly, samples were dehydrated in an ethanol series, cleared in xylene and embedded in paraffin. Paraffin-embedded tissues were sectioned in an automatic tissue processor (Leica Microsystems GmbH, Wetzlar Germany) into 4-5 $\mu \mathrm{m}$-thick slices and subjected to HE staining. For HE staining, samples were stained with hematoxylin for 1-2 min at room temperature. After washing, slices were stained with eosin for 30-60 sec at room temperature. Micrographs under x400 magnification were randomly selected and captured using a light microscope (Nikon Corporation, Tokyo, Japan).

Determination of serum alanine aminotransferase levels. Serum was obtained from blood samples by centrifugation at $3,000 \mathrm{x} \mathrm{g}$ for $10 \mathrm{~min}$ at $4^{\circ} \mathrm{C}$ and was evaluated for serum alanine aminotransferase (ALT) activity using a commercial kit (cat. no. C009-1) according to the manufacturer's protocol (Nanjing Jiancheng Bioengineering Institute, Nanjing, China).

Western blot analysis. A total of $200 \mu \mathrm{g}$ liver tissue was removed from each mouse in the study groups and ground in liquid nitrogen. Protein was extracted using lysis buffer containing $50 \mathrm{mM}$ Tris- $\mathrm{HCl}(\mathrm{pH} 7.5), 150 \mathrm{mM} \mathrm{NaCl}, 1 \%$ Triton X-100, $1 \%$ sodium deoxycholate, $0.1 \%$ SDS, $1 \%$ NP-40, $1 \mu \mathrm{g} / \mathrm{ml}$ leupeptin, $1 \mu \mathrm{g} / \mathrm{ml}$ aprotinin and $1 \mathrm{mM}$ PMSF. The protein concentration was determined using Coomassie Brilliant Blue assay (Fluka Chemie GmbH, Buchs, Switzerland). Proteins were denatured by boiling and $40 \mu \mathrm{g}$ denatured proteins were loaded on and separated by $8 \%$ (for

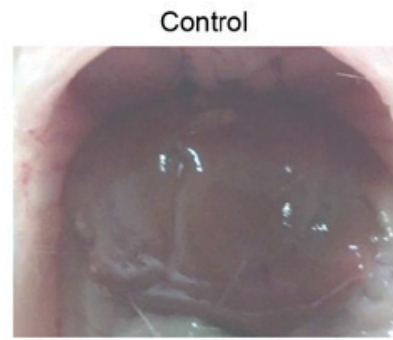

3-h liver injury

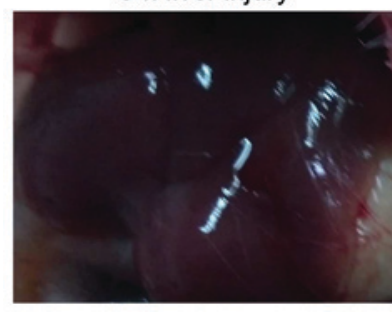

Figure 1. Gross examination of livers obtained from mice in study groups. Images represent control, $\mathrm{n}=8$; 1 -h liver injury group, $\mathrm{n}=8$; 3 -h liver injury group, $\mathrm{n}=8$; and 6-h liver injury group, $\mathrm{n}=9$.

Beclin 1) or $12 \%$ (for LC3) SDS-PAGE, prior to transfer to nitrocellulose membranes. Membranes were blocked in 5\% non-fat dry milk in Tris-buffered saline containing Tween-20 (TBS-T; 0.1\% Tween-20) for $2 \mathrm{~h}$ at room temperature, and then incubated with primary antibodies for overnight at $4^{\circ} \mathrm{C}$. Primary antibodies used include rabbit monoclonal anti-LC3 (1:1,000; cat. no. 13118), rabbit monoclonal anti-Beclin-1 (1:1,000; cat. no. 3495), rabbit monoclonal anti-CHOP (1:1,000; cat no. 5554) and rabbit monoclonal anti- $\beta$-actin $(1: 1,000$; cat. no. 4970) antibodies. Following washing, membranes were incubated with goat anti-rabbit HRP-conjugated secondary antibodies $(1: 4,000$; cat no. BA1003) for $2 \mathrm{~h}$. Immunobands were visualized via exposure to an $\mathrm{x}$-ray beam in a dark room, the $\mathrm{x}$-ray film was scanned by a gel imaging system (JD801; Jiangsu JEDA Science-Technology Development Co., Ltd., China), and the densitometric values of the bands were quantified using ImageJ software version 1.47e (National Institutes of Health, Bethesda, MD, USA). The housekeeping protein $\beta$-actin was used as an internal control.

Statistical analysis. Data were analyzed by SPSS software, version 21 (IBM Corp, Armonk, NY, USA) and results are presented as the mean \pm standard error of the mean. Statistical significance was examined using one-way analysis of variance followed by a post hoc least significant difference test for multiple comparisons. $\mathrm{P}<0.05$ was considered to indicate a statistically significant difference.

\section{Results}

LPS/GalN induces liver injury in mice. The present study firstly established a rodent model of liver injury by injecting LPS and GalN intraperitoneally. In the 6-h liver injury group, 1 mouse died of liver failure at $5.5 \mathrm{~h}$ following model establishment and, therefore, blood and tissue samples of this mouse were not collected for analysis. All other study animals survived until analysis. Gross examination of livers revealed a deep red color and a soft, smooth surface in normal livers (Fig. 1). No 


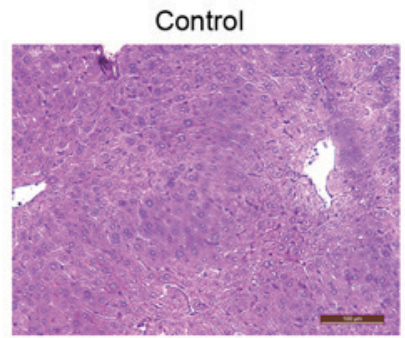

3-h liver injury

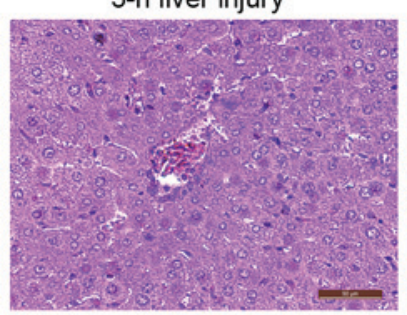

1-h liver injury

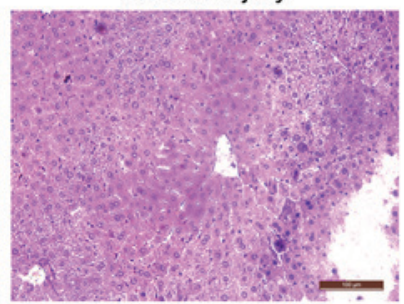

6-h liver injury

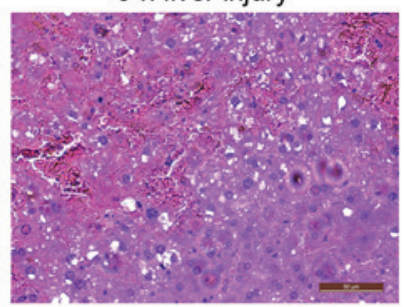

Figure 2. Histological examination of hematoxylin and eosin-stained liver tissues. Representative images of control, $\mathrm{n}=8$; 1 -h liver injury group, $\mathrm{n}=8$; 3 -h liver injury group, $n=8$; and 6 -h liver injury group, $n=9$. Scale bars, $100 \mu \mathrm{m}$.

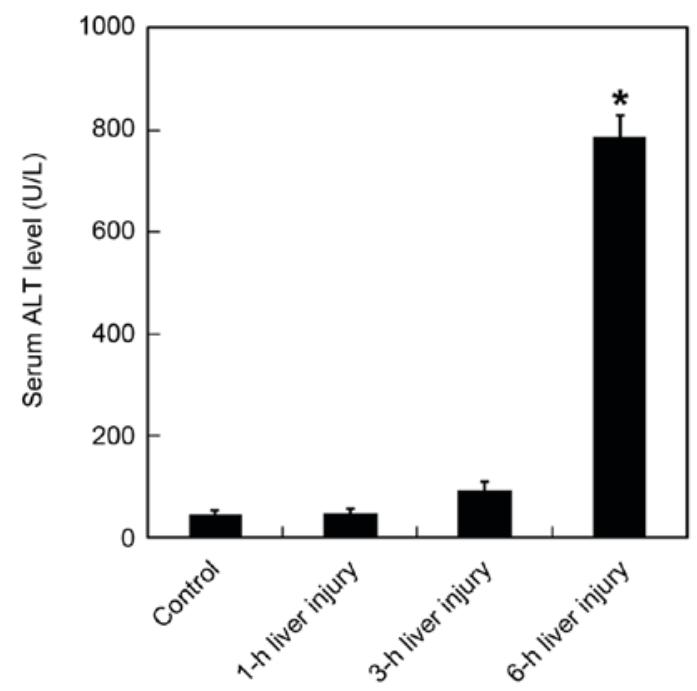

Figure 3. Serum ALT levels increase with LPS-GalN administration. Control group, $\mathrm{n}=8$; 1-h liver injury group, $\mathrm{n}=8$; 3 -h liver injury group, $\mathrm{n}=8$; and $6-\mathrm{h}$ liver injury group, $\mathrm{n}=9$. Mean ALT concentration was calculated. ${ }^{*} \mathrm{P}<0.001$ vs control. LPS, lipopolysaccharide; GalN, D-galactosamine; ALT, alanine aminotransferase.

obvious difference in gross appearance of liver between 1-h and 3-h liver injury groups and the control group was evident. However, livers in the 6-h liver injury group appeared dark red and had a slightly roughened surface. HE-stained liver specimens did not manifest any histological abnormalities in control, 1-h, or 3-h liver injury mice (Fig. 2); however, evident histological alterations with irregularly distributed hepatocytes and abnormal cell morphology were observed in liver tissues of the 6-h liver injury mice. In accordance with these observations, the serum ALT level was significantly elevated at $6 \mathrm{~h}$ following LPS and GalN administration (786.93 $\pm 51.55 \mathrm{U} / 1$; $\mathrm{P}<0.001$ compared with control), although no significant difference was detected in the 1-h or 3-h liver injury groups compared with control (control, 46.23 \pm 8.17 ; 1-h liver injury,
48.07 \pm 9.33 ; 3-h liver injury, 93.49 \pm 16.37 U/1; Fig. 3). These results demonstrated that co-administration of LPS and GalN in mice effectively induced liver injury that evidently occurred $6 \mathrm{~h}$ following drug injection.

LPS/GalN induces autophagy induction during early stage liver injury. In order to understand the potential involvement of autophagy in liver injury, the present study determined the protein expression of autophagy-associated proteins at different stages of liver injury. The conversion from cytosolic (LC3-I) to a lipid-bound form (LC3-II) of LC3 is a reliable biomarker for autophagy induction (13). LC3-II was revealed to be weakly expressed in normal liver tissues (Fig. 4). At $1 \mathrm{~h}$ following liver injury, the LC3-II level was reduced. However, markedly upregulated LC3-II expression was detected at $3 \mathrm{~h}$ following LPS and GalN administration, and the level of LC3-II then declined. Beclin-1 is required for autophagosome formation, which is the initial step of autophagy (13). Consistent with that of LC3-II expression, western blot analysis demonstrated that Beclin-1 expression in liver tissues was reduced at $1 \mathrm{~h}$, whereas it was enhanced at $3 \mathrm{~h}$ following liver injury (Fig. 4). Levels of Beclin 1 reverted to baseline $6 \mathrm{~h}$ following injury (Fig. 4). These findings indicated that liver injury induced by LPS and GalN administration results in initial autophagy inhibition followed by activation, which precedes severe liver injury.

LPS/GalN upregulates CHOP expression during early stage of liver injury. The expression of CHOP is evidence of highly induced ER stress and is, therefore, recognized as a reliable biomarker for the perturbation of the ER (14). It has previously been demonstrated that CHOP contributes to hepatotoxicity (15); therefore, the present study investigated the role of CHOP in LPS/GalN-induced liver damage. Although no significant difference was observed, protein expression of CHOP was slightly reduced in the liver at $1 \mathrm{~h}$ following injury, compared with in healthy liver tissues (Fig. 5). However, a marked upregulation of CHOP was observed at $3 \mathrm{~h}$ following co-administration of LPS and GalN, which reverted to baseline levels at later stages of liver injury (6 h). These data suggested that ER stress occurs concomitantly with autophagy induction, both of which are initiated during early-stage liver injury.

\section{Discussion}

By creating a mouse model of acute liver injury, the present study investigated the potential involvement of autophagy and ER stress in injured liver tissues. The results demonstrated that autophagy-associated proteins (LC3-II, Beclin 1) and the ER-stress-associated protein CHOP were markedly upregulated $3 \mathrm{~h}$ following liver injury, and preceded severe liver injury.

In the present study, acute liver injury in mice was induced by intraperitoneal co-administration of LPS and GalN. LPS constitutes the outer membrane of most gram-negative bacteria, and is essential in mediating endotoxemia (16). Accumulating evidence suggests that intraperitoneal LPS injection induces systemic inflammation and contributes to hepatic dysfunction in rodent studies $(17,18)$. GalN, which is 


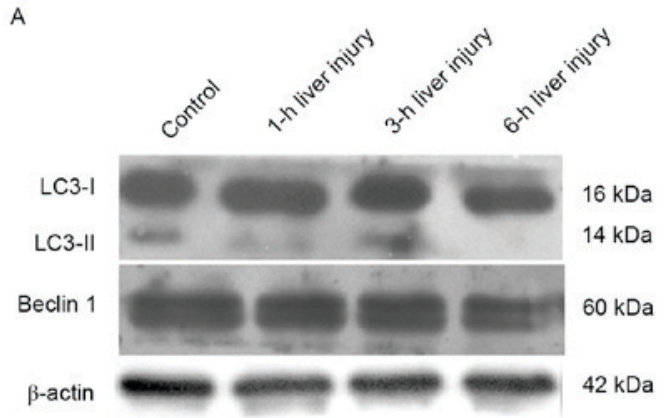

B

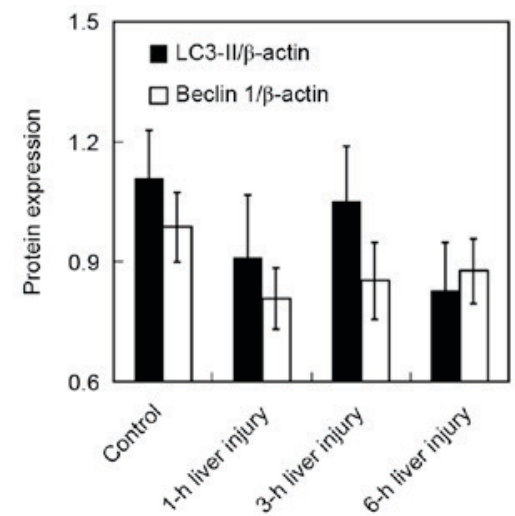

Figure 4. Protein expression of LC3 and Beclin-1 in liver tissues. Tota protein was extracted from liver tissues (control group, $n=8 ; 1$-h liver injury group, $\mathrm{n}=8$; 3-h liver injury group, $\mathrm{n}=8$; and 6-h liver injury group; $\mathrm{n}=9$ ) and expression of LC3-I, LC3-II, and Beclin-1 were determined using western blot analysis. $\beta$-actin was used as an internal control. (A) Representative images and (B) quantification of protein expression normalized to $\beta$-actin LC3, microtubule-associated protein 1 light chain 3.
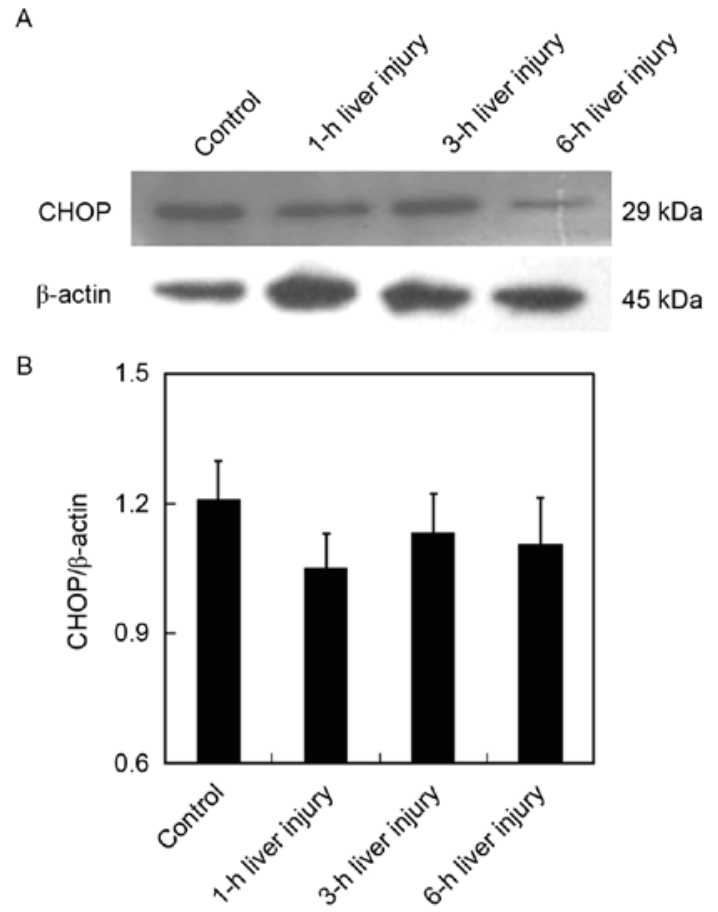

Figure 5. Protein expression of CHOP in liver tissues. Total protein was extracted from liver tissues (control group, $\mathrm{n}=8$; 1-h liver injury group, $\mathrm{n}=8$; 3-h liver injury group, $\mathrm{n}=8$; and 6-h liver injury group, $\mathrm{n}=9$ ) and expression of CHOP determined via western blot analysis. $\beta$-actin was used as an internal control (A) Representative images and (B) quantification of protein expression normalized to $\beta$-actin. CHOP, CCAAT/enhancer binding protein-homologous protein. a hepatocyte-specific inhibitor of RNA synthesis, mediates liver damage through specific induction of uridine triphosphate deficiency in the liver (19). Furthermore, the induction of acute liver injury by GalN administration has been demonstrated in previous studies $(20,21)$. Notably, treatment with GalN sensitizes animals to LPS-induced hepatotoxicity (22); therefore, co-administration of LPS and GalN has a widespread application in inducing acute liver injury in mice. In the present study, LPS-GalN-induced liver injury progressed to severe liver damage with enhanced cell death and elevated serum ALT levels at $6 \mathrm{~h}$ following induction, although no marked ALT or pathological alterations were observed in 1-3 $\mathrm{h}$ following induction. These data are in agreement with a previous report that demonstrated detectable liver injury at $4 \mathrm{~h}$ and significant liver damage at $6 \mathrm{~h}$ following co-treatment with LPS and GalN (23).

Autophagy is a lysosomal-dependent degradative pathway whereby cells digest cytoplasmic components, damaged organelles or long-lived proteins. In the present study, $3 \mathrm{~h}$ following intraperitoneal injection of LPS-GaIN, considerable upregulation of the autophagy-associated proteins LC3-II and Beclin 1 was observed in liver tissues, together with minimal detectable liver injury. Therefore, autophagy may possibly serve as a compensatory mechanism during early liver injury, and induction of autophagy may improve hepatocyte survival by providing energy against an adverse environment. To date, the precise role of autophagy in acute liver injury remains to be fully elucidated. Amir et al (23) demonstrated acceleration of hepatocellular death following LPS-GalN administration in autophagy-associated gene Atg7-deficient mice. Conversely, Li et al (6) revealed that wortmannin, a potent autophagy inhibitor, blocks autophagic activity by alleviating LPS-GalN-induced acute liver injury in mice. These discrepancies may be attributable to the differences between genetic manipulation and pharmacological modulation. Nevertheless, the functional role of autophagy in hepatic injury requires further investigation.

It has previously been demonstrated that ER stress is associated with liver diseases $(24,25)$, and ER stress has been implicated in acetaminophen-induced acute liver failure (26). Furthermore, a marked elevation in protein expression of CHOP, the key molecule in ER stress, has been observed in liver tissues of patients with acute liver failure (10). Sustained hepatocellular ER stress may ultimately lead to hepatocyte death and contribute to liver injury (27). Inhibition of ER stress has been indicated to attenuate liver damage in a rat model of acute liver injury following 90\% hepatectomy (28). Notably, ER stress is a well-characterized inducer of autophagy $(11,12)$, implying the close association between ER stress and autophagy in acute liver injury. The present study detected upregulated CHOP expression with autophagy activation $3 \mathrm{~h}$ following LPS and GalN co-administration. Therefore, LPS-GalN may induce ER stress in liver tissues, subsequently inducing autophagy during early-stage liver damage that may then counter further progression of liver injury. Notably, $6 \mathrm{~h}$ following LPS-GalN injection, autophagy induction and ER stress reverted to baseline levels, indicating this self-protective mechanism may be insufficient for preventing sustained liver damage. These data provide valuable insights into understanding the involvement of 
autophagy and ER stress in the development of acute liver failure.

In summary, the results of the present study reveal that LPS-GalN efficiently induces acute liver injury in mice. Furthermore, ER stress and autophagy induction occur in early-stage liver damage, and this activation of autophagy may have a compensatory role in liver injury.

\section{Acknowledgements}

The present study was supported by Fenyang College, Shanxi Medical University (grant no. 1204).

\section{References}

1. McPhail MJ, Kriese S and Heneghan MA: Current management of acute liver failure. Curr Opin Gastroenterol 31: 209-214, 2015.

2. Kuhla A, Thrum M, Schaeper U, Fehring V, Schulze-Topphoff U, Abshagen $\mathrm{K}$ and Vollmar B: Liver-specific Fas silencing prevents galactosamine/lipopolysaccharide-induced liver injury. Apoptosis 20: 500-511, 2015.

3. Mignon A, Rouquet N, Fabre M, Martin S, Pagès JC, Dhainaut JF, Kahn A, Briand P and Joulin V: LPS challenge in D-galactosamine-sensitized mice accounts for caspase-dependent fulminant hepatitis, not for septic shock. Am J Respir Crit Care Med 159: 1308-1315, 1999.

4. Galanos C, Freudenberg MA and Reutter W: Galactosamine-induced sensitization to the lethal effects of endotoxin. Proc Natl Acad Sci USA 76: 5939-5943, 1979.

5. Malhi H, Gores GJ and Lemasters JJ: Apoptosis and necrosis in the liver: A tale of two deaths?. Hepatology 43 (2 Suppl 1): S31-S44, 2006

6. Li Y, Wang X, Wei Z, Mao H, Gao M, Liu Y, Ma Y, Liu X, Guo C, Zhang $\mathrm{L}$ and Wang X: Pretreatment with wortmannin alleviates lipopolysaccharide/d-galactosamine-induced acute liver injury. Biochem Biophys Res Commun 455: 234-240, 2014.

7. Vicinanza M, Korolchuk VI, Ashkenazi A, Puri C, Menzies FM, Clarke JH and Rubinsztein DC: PI (5)P regulates autophagosome biogenesis. Mol Cell 57: 219-234, 2015.

8. Cursio R, Colosetti P, Codogno P, Cuervo AM and Shen HM: The role of autophagy in liver diseases: Mechanisms and potential therapeutic targets. Biomed Res Int 2015: 480508, 2015.

9. Zhang Q, Li Y, Liang T, Lu X, Zhang C, Liu X, Jiang X, Martin RC, Cheng M and Cai L: ER stress and autophagy dysfunction contribute to fatty liver in diabetic mice. Int J Biol Sci 11: 559-568, 2015

10. Rao J, Zhang C, Wang P, Lu L, Qian X, Qin J, Pan X, Li G, Wang $X$ and Zhang F: C/EBP homologous protein (CHOP) contributes to hepatocyte death via the promotion of ERO1a signalling in acute liver failure. Biochem J 466: 369-378, 2015.

11. Rashid HO, Yadav RK, Kim HR and Chae HJ: ER stress: Autophagy induction, inhibition and selection. Autophagy 11: 1956-1977, 2015
12. Lee WS, Yoo WH and Chae HJ: ER stress and autophagy. Curr Mol Med 15: 735-745, 2015.

13. Feng Y, He D, Yao Z and Klionsky DJ: The machinery of macroautophagy. Cell Res 24: 24-41, 2014.

14. Sano R and Reed JC: ER stress-induced cell death mechanisms. Biochim Biophys Acta 1833: 3460-3470, 2013.

15. Uzi D, Barda L, Scaiewicz V, Mills M, Mueller T, Gonzalez-Rodriguez A, Valverde AM, Iwawaki T, Nahmias Y, Xavier R, et al: CHOP is a critical regulator of acetaminophen-induced hepatotoxicity. J Hepatol 59: 495-503, 2013.

16. Wang X and Quinn PJ: Endotoxins: Lipopolysaccharides of gram-negative bacteria. Subcell Biochem 53: 3-25, 2010.

17. El-Tanbouly DM, Abdelsalam RM, Attia AS and Abdel-Aziz MT: Pretreatment with magnesium ameliorates lipopolysaccharide-induced liver injury in mice. Pharmacol Rep 67: 914-920, 2015.

18. Gao LN, Yan K, Cui YL, Fan GW and Wang YF: Protective effect of Salvia miltiorrhiza and Carthamus tinctorius extract against lipopolysaccharide-induced liver injury. World J Gastroenterol 21: 9079-9092, 2015.

19. Keppler DO, Pausch J and Decker K: Selective uridine triphosphate deficiency induced by D-galactosamine in liver and reversed by pyrimidine nucleotide precursors. Effect on ribonucleic acid synthesis. J Biol Chem 249: 211-216, 1974.

20. Lu Y, Wang WJ, Song YZ and Liang ZQ: The protective mechanism of schisandrin A in d-galactosamine-induced acute liver injury through activation of autophagy. Pharm Biol 52: 1302-1307, 2014

21. Zhang Z, Zhao YC, Cheng Y, Jian GD, Pan MX and Gao Y: Hybrid bioartificial liver support in cynomolgus monkeys with D-galactosamine-induced acute liver failure. World J Gastroenterol 20: 17399-17406, 2014.

22. Alcorn JM, Fierer J and Chojkier M: The acute-phase response protects mice from D-galactosamine sensitization to endotoxin and tumor necrosis factor-alpha. Hepatology 15: 122-129, 1992.

23. Amir M, Zhao E, Fontana L, Rosenberg H, Tanaka K, Gao G and Czaja MJ: Inhibition of hepatocyte autophagy increases tumor necrosis factor-dependent liver injury by promoting caspase- 8 activation. Cell Death Differ 20: 878-887, 2013.

24. Dara L, Ji C and Kaplowitz N: The contribution of endoplasmic reticulum stress to liver diseases. Hepatology 53: 1752-1763, 2011.

25. Malhi H and Kaufman RJ: Endoplasmic reticulum stress in liver disease. J Hepatol 54: 795-809, 2011.

26. Tai M, Zhang J, Song S, Miao R, Liu S, Pang Q, Wu Q and Liu C: Protective effects of luteolin against acetaminophen-induced acute liver failure in mouse. Int Immunopharmacol 27: 164-170, 2015.

27. Han CY, Lim SW, Koo JH, Kim W and Kim SG: PHLDA3 overexpression in hepatocytes by endoplasmic reticulum stress via IRE1-Xbp1 s pathway expedites liver injury. Gut 65: 1377-1388, 2016.

28. Jia C, Dai C, Bu X, Peng S, Xu F, Xu Y and Zhao Y: Co-administration of prostaglandin $\mathrm{E} 1$ with somatostatin attenuates acute liver damage after massive hepatectomy in rats via inhibition of inflammatory responses, apoptosis and endoplasmic reticulum stress. Int J Mol Med 31: 416-422, 2013. 